Acta Cryst. (1953). 6, 817

\title{
A New Method of Calculating the Scale Factor in Structure Analysis
}

\author{
By Gopinath KaRtha \\ Department of Physics, Indian Institute of Science, Bangalore 3, India
}

(Received 22 April 1953 and in revised form 8 June 1953)

\begin{abstract}
A new method is suggested for obtaining the absolute structure factors from a set of relative intensities. It differs from the method of Wilson in that it does not depend on any statistical result. Formulae are obtained relating the sum of the intensities of all the reflexions with certain integrals involving the electron-density distribution in the atoms. The method is applicable both to two- and three-dimensional data, but in practice it is more useful in the former case. The method makes use of the available structure-factor tables, and has been verified to be valid in a number of cases where the scale factor is known. For the purpose of applying the method to unknown cases, tables of the required integrals are given for some of the commoner atoms which occur in organic crystals.
\end{abstract}

\section{Introduction}

In crystal-structure analysis, the observed intensities of the various reflexions $(h k l)$, after the necessary corrections, yield a set of numbers $I_{h k l}$, which are proportional to $\left|F_{h k l}\right|^{2}$, the constant of proportionality, the scale factor $c$, being given by the equation

$$
c I_{h k l}=\left|F_{h k l}\right|^{2} .
$$

The only direct method of obtaining the scale factor, without having to measure the absolute intensities of some of the reflexions or comparing them with those of a standard crystal, is that due to Wilson (1942). The method is based on the statistical result that in a small range of $\sin \theta \mid \lambda$ the mean of $\left|F_{h k l}\right|^{2}$ is equal to the sum of the mean squares of the atomic scattering factors of the atoms in the unit cell for the same range. In many crystals, the number of reflexions in a suitable chosen range may be quite small (of the order of 10-20), so that statistical methods cannot be strictly applied to them.

The present paper describes a method which makes use of an exact relationship between the sum of the squared structure amplitudes of all reflexions and a certain integral involving the electron-density distribution of the atoms in the unit cell, a relationship which does not assume any statistical results. However, it requires a knowledge of the approximate temperature factor, but, if this is known, the method is much simpler to apply than Wilson's method and yields more accurate results. The method has been tested in a number of known cases and the results obtained are very satisfactory in every case.

\section{The fundamental equation}

The electron density $\varrho(\mathbf{r})$ at a point $\mathbf{r}$ inside the unit cell may be represented by the Fourier series

$$
\varrho(\mathbf{r})=\frac{1}{V} \sum_{H} F_{H} \exp \left[2 \pi i\left(\mathbf{B}_{H} \cdot \mathbf{r}\right)\right],
$$

where $\Sigma_{H}$ denotes summation with respect to the triplet of integers $h, k, l$; and $\mathbf{B}_{H}$ is the vector $h \mathbf{a}^{*}+k \mathbf{b}^{*}+l \mathbf{c}^{*}$ in the reciprocal space. $V$ is the volume of the unit cell.

By squaring (2) and integrating over the unit cell we have

$\int_{\nabla} \varrho^{2}(\mathbf{r}) d V=\frac{1}{V^{2}} \sum_{H} \sum_{H^{\prime}} F_{H} F_{H^{\prime}} \int_{V} \exp \left[2 \pi i\left(\mathbf{B}_{H+H^{\prime}} . \mathbf{r}\right)\right] d V$.

Since the integral on the right-hand side is equal to $V \delta_{H,-H^{\prime}}$, we have

$$
\int_{V} \varrho^{2} d V=\frac{1}{V} \sum_{H} F_{H} F_{-H} .
$$

By the application of Friedel's Law, $F_{-H}=F_{H}^{*}$, this reduces to

$$
\int_{V} \varrho^{2} d V=\frac{1}{V} \sum_{H} F_{H} F_{H}^{*}=\frac{1}{V} \sum_{h} \sum_{-\infty}^{\infty} \sum_{l}\left|F_{h k l}\right|^{2},
$$

a result which has been given earlier by Hettich (1935). Thus we get the following equation for the scale factor, $c$ :

$$
c=V \int_{V} \varrho^{2} d V / \sum_{h} \sum_{k}^{\infty} \sum_{-\infty} I_{h k l} .
$$

Similarly, in the two-dimensional projection along the $c$-axis we have

$$
c=A \int_{A} \sigma^{2} d A / \sum_{h}^{\infty} \sum_{k}^{\infty} I_{h k 0},
$$

where $\sigma$ is the projected electron density on the side of the unit cell of area $A$.

Now, if we assume that the electron density in the 
unit cell is obtained by the superposition of the electron-density distributions of individual atoms, and that there is very little overlap of the distributions of different atoms, then we may write

$$
\int_{V} \varrho^{2}(\mathbf{r}) d V=\sum_{S=1}^{N} \int_{v} \varrho_{S}^{2} d v
$$

where $N$ is the number of atoms in the unit cell. The corresponding equation

$$
\int_{\Delta} \sigma^{2}(\mathbf{r}) d A=\sum_{S=1}^{N} \int_{a} \sigma_{S}^{2} d a
$$

holds if the atoms are all well resolved in the projection. In three dimensions, the error introduced by this assumption is practically negligible.

Now, it is possible to represent the two integrals $\int e_{S}^{2} d v$ and $\int \sigma_{S}^{2} d a$ in terms of certain integrals of the atomic structure factors. If $\varrho_{S}(\mathbf{r})$ is the electron density of an atom $s$ at distance $\mathbf{r}$ from its centre, and $f_{S}(\xi)$ is its atomic scattering factor for a value of $\xi=4 \pi \sin \theta / \lambda=2 \pi|b|=2 \pi b$, then by Fourier inversion theorem we have

Hence

$$
\varrho_{S}(\mathbf{r})=\int_{v^{*}} f_{S}(\xi) \exp [2 \pi i(\mathbf{b} . \mathbf{r})] d v^{*}
$$

$\int_{v} \varrho_{S}^{2}(\mathrm{r}) d v$

$=\int_{v} \int_{v^{*}} \int_{v^{*}} f_{S}\left(\xi_{1}\right) f_{S}\left(\xi_{2}\right) \exp \left[2 \pi i\left\{\left(\mathbf{b}_{1}+\mathbf{b}_{2}\right) \cdot \mathbf{r}\right\}\right] d v d v_{1}^{*} d v_{2}^{*}$.

Since $f$ is a symmetric function and

$$
\iint_{v} \exp \left[2 \pi i\left\{\left(\mathbf{b}_{1}+\mathbf{b}_{2}\right) \cdot \mathbf{r}\right\}\right] d v d v^{*}=\delta\left(\mathbf{b}_{1}+\mathbf{b}_{2}\right),
$$

we finally obtain

$$
\begin{aligned}
\int_{v} e_{S}^{2}(\mathbf{r}) d v & =\int_{v^{*}} f_{S}^{2}(\xi) d v^{*} \\
& =4 \pi \int_{0}^{\infty} f_{S}^{2}(\xi) b^{2} d b \\
& =\left(\frac{1}{2 \pi^{2}}\right) \int_{0}^{\infty} \xi^{2} f_{S}^{2}(\xi) d \xi .
\end{aligned}
$$

In a very similar manner we may obtain the expression

$$
\int_{a} \sigma_{S}^{2}(\mathbf{r}) d a=\left(\frac{1}{2 \pi}\right) \int_{0}^{\infty} \xi f_{S}^{2}(\xi) d \xi
$$

for the two-dimensional case.

\section{Application of the method}

In determining the scale factor, using equations (5) and (6), one could either use directly the electrondensity integrals of the individual atoms, or obtain them, using (9) and (10), from their tabulated scat- tering factors. The contribution to the total integral by different atoms could be calculated separately for each atom and for different temperature factors.

Although the electron-density distributions of a large number of atoms have been calculated on the basis of modern wave mechanical theory (Hartree, 1928) these are not to be had in explicit algebraic forms suitable for integration analytically. Instead, empirical formulae have been proposed to represent the distribution. Thus, it has been suggested (see Booth, 1945) that charge distributions obtained by Fourier syntheses could be very closely represented by expressions of the form

$$
\varrho_{S}(r)=A_{S} \exp \left[-P_{S} r^{2}\right],
$$

and this form, with $P=4 \cdot 689$, has been widely used by Booth (1945) in many studies. Obviously the value of the exponent as well as the form of the function would depend on the temperature factor, and it is not clear how the effect of these could be simply incorporated in the above expression. Further, an atom with a Gaussian distribution would give an atomic scattering factor curve which is also Gaussian. The author's examination of a series of atomic scattering factor curves with different temperature factors showed that the fall of scattering factor with $\sin \theta / \lambda$ was much larger, for small values of $\sin \theta / \lambda$, and much smaller, for large values of $\sin \theta / \lambda$, than what would be represented by a Gaussian form. In fact, the scattering-factor values given by Robertson (1935) for hydrocarbons at room temperature were found to be represented with remarkable accuracy by an analytical expression not of the Gaussian form but of the form $R \exp [-b \xi]$. In view of these difficulties this direct method was not pursued further.

However, the atomic integrals $\left[\int \varrho_{S}^{2} d v\right.$ and $\left.\int \sigma_{\mathrm{S}}^{2} d a\right]$ can be readily calculated from equations (9) and (10), using the tabulated values of the atomic scattering factors and the known value of the temperature factor. In equations (5) and (6), the $I_{h k l}$ 's in the denominator would refer to the experimental values obtained without applying the temperature correction, so that the integrals in the numerator should also refer to the same temperature. The effect of temperature can readily be incorporated in equations (9) and (10), for we know that it changes $f(\xi)$ to $f(\xi) \exp \left[-B(\xi / 4 \pi)^{2}\right]$, which we shall denote by $f_{B}(\xi)$. Now the value of $f(\xi)$ for various values of $\xi$ are available from standard tables for different atoms, and therefore a table of integrals on the right-hand side of (9) and (10) can be prepared for a number of atoms and for various temperature factors, $B$. Since the scale factor would in general have to be determined only for reflexions in a zone, the values of $\int \sigma_{s}^{2} d a$ calculated as described above are given in Table 1 for the atoms $\mathrm{C}, \mathrm{H}, \mathrm{O}$, $\mathrm{N}, \mathrm{S}, \mathrm{C}, \mathrm{Ni}$ and $\mathrm{Cu}$ for various temperature factors.

In applying the method, the following procedure is to be followed: 
Table 1. Values of $\left(\frac{1}{2 \pi}\right) \int_{0}^{\infty} \xi f_{B}^{2}(\xi) d \xi$ for some atoms

\begin{tabular}{|c|c|c|c|c|c|c|c|c|c|c|c|c|c|c|c|}
\hline $2 B$ & 0.5 & 1.0 & 1.5 & $2 \cdot 0$ & $2 \cdot 5$ & $3 \cdot 0$ & $3 \cdot 5$ & $4 \cdot 0$ & $4 \cdot 5$ & $5 \cdot 0$ & $5 \cdot 5$ & $6 \cdot 0$ & 6.5 & $7 \cdot 0$ & $10 \cdot 0$ \\
\hline $\mathrm{H}$ & $0 \cdot 37$ & 0.37 & 0.35 & $0 \cdot 35$ & 0.34 & $0 \cdot 34$ & 0.33 & 0.33 & 0.32 & 0.31 & 0.31 & 0.30 & 0.30 & $0 \cdot 29$ & 0.27 \\
\hline C & 38.8 & $32 \cdot 3$ & 26.5 & 23.5 & $2 l \cdot 3$ & $19 \cdot 7$ & 18.4 & $17 \cdot 3$ & $16 \cdot 3$ & $15 \cdot 6$ & 14.9 & $14 \cdot 4$ & $13 \cdot 8$ & $13 \cdot 4$ & $11 \cdot 3$ \\
\hline $\mathrm{N}$ & $56 \cdot 1$ & $45 \cdot 4$ & $39 \cdot 5$ & $35 \cdot 5$ & $32 \cdot 7$ & $30 \cdot 6$ & 28.9 & $27 \cdot 5$ & $26 \cdot 2$ & $25 \cdot 2$ & $24 \cdot 3$ & $23 \cdot 4$ & $22 \cdot 7$ & $22 \cdot 0$ & 18.9 \\
\hline $\mathrm{O}$ & $82 \cdot 4$ & $68 \cdot 3$ & $60 \cdot 6$ & $55 \cdot 4$ & $51 \cdot 6$ & $48 \cdot 6$ & $46 \cdot I$ & $44 \cdot 1$ & $42 \cdot 4$ & $40 \cdot 8$ & $39 \cdot 4$ & $38 \cdot 2$ & $37 \cdot 1$ & $36 \cdot 1$ & $31 \cdot 3$ \\
\hline $\mathrm{Na}$ & 197 & 167 & 150 & 137 & 127 & 119 & 112 & 107 & 101 & 97 & 93 & 89 & 86 & 83 & 69 \\
\hline $\mathbf{S}$ & 486 & 396 & 342 & 303 & 274 & 251 & 232 & 217 & 203 & 192 & 182 & 173 & 165 & 158 & 127 \\
\hline $\mathrm{Cl}$ & 561 & 454 & 388 & 343 & 309 & 283 & 261 & 243 & 228 & 215 & 204 & 194 & 185 & 177 & 143 \\
\hline $\mathrm{Ni}$ & 1780 & 1401 & 1194 & 1058 & 958 & 881 & 818 & 765 & 721 & 682 & 648 & 618 & 590 & 566 & 457 \\
\hline $\mathrm{Cu}$ & 2033 & 1617 & 1387 & 1234 & 1121 & 1032 & 960 & 899 & 847 & 802 & 762 & 727 & 695 & 666 & 538 \\
\hline
\end{tabular}

Table 2. Results of application of the structure-factor method to some known cases

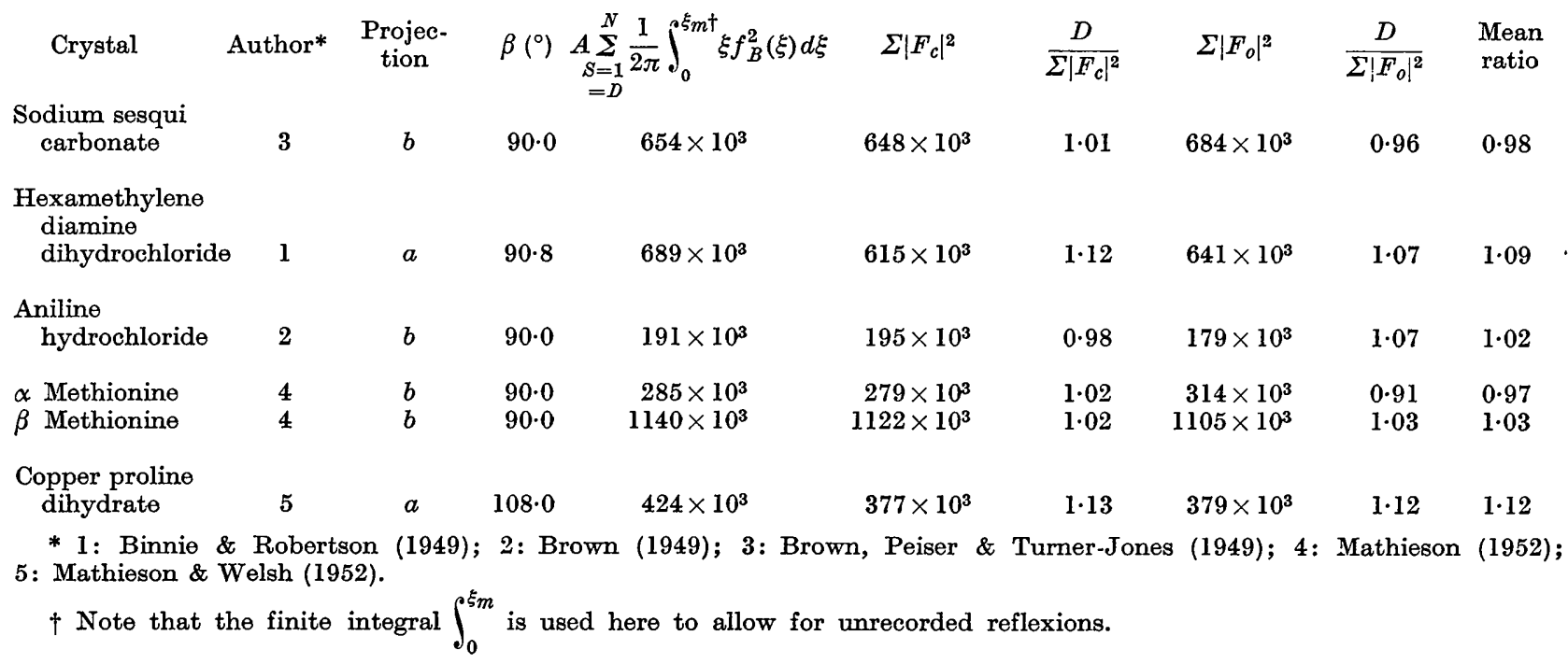

The temperature factor $B$ may be approximately obtained from a knowledge of the melting point or from the value of $\sin \theta / \lambda$ at which the average intensity of the reflexions reduces to, say, $1 / 1000$ of the maximum value. That the exact value of $B$, provided it is not very small, is not very important is found from the value given in Table 1 . The value of the $\varrho^{2}$ or $\sigma^{2}$ integrals are then obtained from the table. Next find

$$
\sum_{h} \sum_{k} \sum_{l} I_{h k l} \text { (or } \sum_{h} \sum_{k} I_{h k 0} \text { etc.) }
$$

from the observed values of intensities by finding the sum of all the observed intensities, the respective multiplicities being taken into account. The ratio of the two gives the required scale factor. However, it must be remembered that $I_{\mathbf{0 0 0}}$ is not directly observed. Consequently, the corresponding quantity in the atomic integral, namely $\left|F_{000}\right|^{2}$, should be omitted. Obviously $\left|F_{000}\right|^{2}=Z^{2}$, where $Z$ is the total number of electrons in the unit cell. Hence the scale factor in the two-dimensional case is given by equations of the form

$$
c=A\left[\sum_{S=1}^{N} \int \sigma_{S}^{2} d a\right]-Z^{2} / \sum_{h} \sum_{k} I_{h k 0},
$$

where $I_{000}$ is omitted in summing the intensity values.

The finiteness of the series $\Sigma I$ could be allowed for directly by calculating the finite integrals $\int_{0}^{\xi_{m}}$ where $\xi_{m}$ is the maximum value of $4 \pi \sin \theta / \lambda$ up to which the reflexions have been observed. We are justified in doing this because the reflexion density is very large for large values of $\xi$ so that in these regions the mean intensity could be assumed to be the sum of the squares of the scattering factors of the various atoms in the unit cell. Further, any error due to this assumption is very small since $\Sigma I$ for values of $\xi$ larger than $\xi_{m}$ will usually be very small for most light atoms present in organic crystals provided the maximum value of $\xi_{m}$ obtainable by $\mathrm{Cu} K \alpha$ radiation is used.

\section{Test of the validity of the method in known cases}

To show the extent to which the assumptions of this method are justified, the method has been applied to a number of crystal projections where $\left|F_{h k l}\right|$ values have been given on an absolute scale so that $c=1$ in these cases. The results are given in Table 2 ; both $F_{\circ}$ and $F_{c}$ values are used in obtaining the sum $\Sigma I$. 
It is seen that the agreement is satisfactory, the values being correct to within $10 \%$. This could be regarded as appreciably better than Wilson's method, where the value of $c$ was found to vary within the limit 0.8-1.5 whereas the correct value was expected to be unity. It is of interest to note that even though we have assumed that the plane of projection is perpendicular to the direction of projection in evaluating the $\sigma^{2}$-integral, the agreement is good even in case of monoclinic crystals projected along axes other than the $b$ axis. This has been so in the case of hexamethylenediamine dihydrochloride and copper proline dihydrate, where the plane of projection made angles $90.8^{\circ}$ and $108^{\circ}$ respectively with the direction of projection. It is easily seen that the actual $\sigma^{2}$-integral in the case of such projections will be less than the value in the case of normal projections, and hence $c$ values obtained by the above method are liable to be larger than the true value as the angle $\beta$ differs more and more from the value $90^{\circ}$.

In the case of unknown crystals, even if tables of the integrals

$$
\int_{0}^{\xi m} \xi^{2} f_{B}(\xi) d \xi \text { or } \int_{0}^{\xi m} \xi f_{B}^{2}(\xi) d \xi
$$

are not available they can be readily calculated from the tables of atomic scattering factors. An extended table of these factors for the lighter atoms, given by Viervoll \& Ögrim (1949), has been used by the author in preparing the Table 1 .

\section{The advantages of the present method}

The equation

$$
\frac{1}{V} \sum_{h} \sum_{-\infty}^{\infty} \sum_{l}\left|F_{h k l}\right|^{2}=\sum_{S=1}^{N}\left(\frac{1}{2 \pi^{2}}\right) \int_{0}^{\infty} \xi^{2} f^{2}(\xi) d \xi,
$$

and the counterpart in two dimensions, which form the basis of the method of calculating the scale factor proposed in this paper, are exact relations. Therefore, when the temperature factor is known, this provides an accurate method of determining the scale factor.

Now, it can be shown* (Sayre, 1951) that

$$
\left(\frac{1}{2 \pi^{2}}\right) \int_{0}^{\infty} \xi^{2} f_{S}^{2}(\xi) d \xi=\frac{1}{V} \sum_{h} \sum_{k}^{\infty} \sum_{l} f_{S}^{2}(h k l),
$$

so that we get the relationship

$$
\sum_{h} \sum_{\substack{k \\-\infty}}^{\infty} \sum_{l}\left|F_{h k l}\right|^{2}=\sum_{h} \sum_{\substack{k \\-\infty}}^{\infty} \sum_{l}\left\{\sum_{S=1}^{N} f_{S}^{2}(h k l)\right\} .
$$

Equation (14) is an exact result which holds for atoms whose electron density falls to zero in a comparatively

* The author is grateful to Dr Cochran for pointing this out. short distance. Obviously, however, this form is not suitable for computational purposes since, for each crystal, the sum on the right-hand side will have to be evaluated and the number of terms occurring is very large (approximately $N \times H \times K \times L)$. In (13), however, only a small number (equal to $N$, the number of atoms in the unit cell) of integrals have to be added, once the preliminary tables of integrals have been prepared. The same tables could be used for all crystals.

If, however, we confine our attention to a small range of $\sin \theta / \lambda$, then (14) leads to the statistical result of Wilson as a special case, namely

$$
\left\langle\left|F_{h k l}\right|^{2}\right\rangle_{\mathrm{av} .}=\sum_{S=1}^{N} \bar{f}_{S}^{2}
$$

The only advantage of the statistical method over the exact method appears to be that it can be used to determine both the scale factor and the temperature factor. However, as can be seen from Table 1, the exact method is not very sensitive to variations of the temperature factor. On the other hand, it has the advantage that it does not depend on any statistical result which may not lead to accurate results. Further, the present method has the advantage that once the tables of the atomic integrals are prepared, the calculation of the scale factor is much simpler than in Wilson's method. On the other hand, the present method, as it stands now, could be used directly only for determination of scale factor of zero-level photographs by using equations of the form $A \int \sigma^{2} d A=$ $\Sigma \Sigma\left|F_{h k 0}\right|^{2}$, whereas Wilson's method could be used for any layer photographs since his averaging results hold for any large number of reflexions, provided the choice of reflexions is absolutely random.

The author wishes to express his thanks to Prof. R. S. Krishnan for his kind interest and to Dr G. N. Ramachandran for valuable guidance and help.

\section{References}

Binnte, W. P. \& Robertson, J. M. (1949). Acta Cryst. 2, 180.

Booth, A. D. (1945). Proc. Roy. Soc. A, 188, 77.

Brown, C. J. (1949). Acta Cryst. 2, 228.

Brown, C. J., Peiser, H. S. \& Turner-Jones, A. (1949). Acta Cryst. 2, 167.

Hartree, D. R. (1928). Proc. Camb. Phil. Soc. 24, 111. Hettich, A. (1935). Z. Krystallogr. 91, 154.

Mathieson, A. McL. (1952). Acta Cryst. 5, 332.

Mathieson, A. McL. \& Welsh, H. K. (1952). Acta Cryst. $5,599$.

Robertson, J. M. (1935). Proc. Roy. Soc. A, 150, 121. SAYRe, D. (1951). Acta Cryst. 4, 362.

VIERvold, H. \& ÖGRIM, O. (1949). Acta Cryst. 2, 277. Wilson, A. J. C. (1942). Nature, Lond. 150, 152. 